\title{
Evaluation of a microarray-hybridization based method applicable for discovery of single nucleotide polymorphisms (SNPs) in the Pseudomonas aeruginosa genome
}

\author{
Andreas Dötsch, Claudia Pommerenke, Florian Bredenbruch, Robert Geffers \\ and Susanne Häussler
}

Helmholtz Centre for Infection Research, Inhoffenstraße 7, 38124 Braunschweig, Germany

E-mail: Andreas Dötsch - andreas.doetsch@helmholtz-hzi.de; Claudia Pommerenke - claudia.pommerenke@helmholtz-hzi.de; Florian Bredenbruch - florian.bredenbruch@helmholtz-hzi.de; Robert Geffers - robert.geffers@helmholtz-hzi.de;

Susanne Häussler* - susanne.haeussler@helmholtz-hzi.de;

*Corresponding author

Published: 19 January 2009

BMC Genomics 2009, 10:29 doi: 10.1186/1471-2164-10-29
Received: 23 July 2008

Accepted: 19 January 2009

This article is available from: http://www.biomedcentral.com/I47I-2/64/I0/29

(C) 2009 Dötsch et al; licensee BioMed Central Ltd.

This is an Open Access article distributed under the terms of the Creative Commons Attribution License (http://creativecommons.org/licenses/by/2.0), which permits unrestricted use, distribution, and reproduction in any medium, provided the original work is properly cited.

\begin{abstract}
Background: Whole genome sequencing techniques have added a new dimension to studies on bacterial adaptation, evolution and diversity in chronic infections. By using this powerful approach it was demonstrated that Pseudomonas aeruginosa undergoes intense genetic adaptation processes, crucial in the development of persistent disease. The challenge ahead is to identify universal infection relevant adaptive bacterial traits as potential targets for the development of alternative treatment strategies.
\end{abstract}

Results: We developed a microarray-based method applicable for discovery of single nucleotide polymorphisms (SNPs) in P. aeruginosa as an easy and economical alternative to whole genome sequencing. About $50 \%$ of all SNPs theoretically covered by the array could be detected in a comparative hybridization of PAOI and PAI 4 genomes at high specificity $(>0.996)$. Variations larger than SNPs were detected at much higher sensitivities, reaching nearly $100 \%$ for genetic differences affecting multiple consecutive probe oligonucleotides. The detailed comparison of the in silico alignment with experimental hybridization data lead to the identification of various factors influencing sensitivity and specificity in SNP detection and to the identification of strain specific features such as a large deletion within the PA4684 and PA4685 genes in the Washington Genome Center PAOI.

Conclusion: The application of the genome array as a tool to identify adaptive mutations, to depict genome organizations, and to identify global regulons by the "ChIP-on-chip" technique will expand our knowledge on $P$. aeruginosa adaptation, evolution and regulatory mechanisms of persistence on a global scale and thus advance the development of effective therapies to overcome persistent disease.

\section{Background}

Pseudomonas aeruginosa is a very versatile bacterial organism that has a unique capability to thrive and survive in a great variety of habitats. The bacterium is ubiquitously found in aquatic and terrestic environments and has evolved as an important opportunistic pathogen that causes serious infections in plants, insects and vertebrates $[1,2]$. In the human host $P$. aeruginosa is mainly a nosocomial pathogen feared for entailing acute pneumonia and sepsis accompanied with 
very high mortality rates $[3,4]$. Moreover, $P$. aeruginosa is a dominant bacterial pathogen that causes chronic infections in patients with burns or suffering from cystic fibrosis (CF) [5-7]. Most of the CF patients acquire $P$. aeruginosa from the environment during their early life-time [8] and develop a chronic P. aeruginosa lung infection which largely determines the fate and prognosis in these patients. A very characteristic finding is that the same bacterial lineage usually persists for years or even decades in the chronically infected CF lung and cannot be eradicated even by intensified anti-pseudomonas therapy [9]. Thus, in order to overcome chronic persistent disease, novel anti-microbial treatment strategies are desperately needed. For that purpose, it seems to be essential to gain detailed insight into the molecular mechanisms that underlie bacterial adaptation processes to the environment of the cystic fibrosis lung, which results in the evolution of a protected and persistent bacterial population. Recently Smith at al. [10] have conducted a very powerful approach to gain global information on $P$. aeruginosa adaptation to the chronically infected CF lung. They performed whole genome sequencing of an early and a late $P$. aeruginosa $\mathrm{CF}$ isolate and could show that many single-base changes accumulated in the late isolate that were obviously advantageous for the life within the host. Intriguingly, these mutations caused loss of functions used by bacteria to invade and injure the host, indicating that they may become a burden once the chronic infection has become established. The work by Smith et al. [10] has opened a window to complex questions of bacterial adaptation and evolution during chronic infections. The great challenge now is to expand the search for adaptive mutations and to validate the findings. Furthermore a more detailed analysis whether evolution produces one adapted strain or whether it produces a diverse community of infecting bacteria is desirable.

In this study we have designed a $P$. aeruginosa Affymetrix custom made "tiling" array (PATA1) composed of approximately 250.000 25-mer oligonucleotides that due to the large chromosome - depicts approximately $85 \%$ of the PAO 1 genome. To our knowledge, PATA1 is the first tiling array targeting a complete Pseudomonas genome. The probes of a tiling array are more or less equally distributed across the genome instead of using a small subset of probes targeting e.g. ORFs like the Affymetrix PAO1 GeneChip ${ }^{\circledast}$ or other available DNA microarrays. Therefore, tiling arrays produce unbiased data on the whole chromosome. The method has been successfully applied to detect mutations in Helicobacter pylori [11]. We evaluated whether with a microarrayhybridisation based method this array is capable of identifying genome variations in $P$. aeruginosa as a costeffective alterative to whole genome sequencing. By comparative hybridization of chromosomal DNA of a PAO1 and PA14 strain, we clearly demonstrate that our custom made PATA1 array efficiently detects inter-strain genetic variations even at the level of single nucleotide polymorphisms (SNPs) and can be used as a highly sophisticated tool for the identification of the $P$. aeruginosa genome organization.

\section{Methods \\ Organism \& Culturing}

The $P$. aeruginosa strains used for the microarray experiments presented in this study were PAO1 (parental strain of the Washington Transposon Mutant Library, [12]) and PA14 (obtained from the Ausubel Lab). $P$. aeruginosa DSM1707 was used as a reference strain to test for the presence of the PA4685 deletion. Cultures were grown in brain-heart infusion (BHI) medium at $37^{\circ} \mathrm{C}$ in shaking glass flasks or tubes at $180 \mathrm{rpm}$.

\section{DNA preparation and Microarray hybridization}

Cell samples were harvested from $1 \mathrm{~mL}$ of $12 \mathrm{~h}$ stationary phase liquid cultures. Genomic DNA was isolated using the DNeasy Blood \& Tissue Kit (Qiagen, Hamburg, Germany). Cell lysates were treated with RNase I (Qiagen) to prevent accidental carryover of RNA to the microarray. Genomic DNA was partially digested with DNase I (Amersham Biosciences, Piscataway, NJ) to a fragment size of $50-250 \mathrm{bp}$, confirmed by gel electrophoresis, and fragments were labeled at the 3 '-ends with biotin-ddUTP (Roche Diagnostics, Indianapolis, IN) using Terminal deoxynucleotidyl transferase (Roche).

For each sample 4 - $5 \mu \mathrm{g}$ of labeled DNA fragments were hybridized to an identical lot of PATA1 array for 16 hours at $50^{\circ} \mathrm{C}$.

After hybridization the GeneChips were washed, stained with SA-PE and read using an Affymetrix GeneChip fluidic station and scanner according to Affymetrix standard protocols (Affymetrix, Santa Clara, CA).

\section{Validation of a I kb deletion at PA4685 by PCR}

Two sets of primers were designed to test genomic DNA for presence of the $1 \mathrm{~kb}$ deletion identified from microarray data. Primers A1 (5'-TCG CAG GTC GAG AGC TAC GT-3') and A2 (5'-ATG CGT CAG CCT CCT GTT GC-3') are placed outside the suspected region while B1 (5'-GCT GCC GGA CCT CAT GCA AT-3') and B2 (5'-TCG CGG TGG CTG ATG TGG TA-3') span a sequence inside this region. DNA polymerase GoTaq (Promega, Madison, WI) was used for PCR. 


\section{Analysis of tiling array data}

Analysis of microarray data was performed using the Affymetrix GCOS 1.4 to generate the raw data files (cel data).

The raw data files were further analyzed using 'Tiling Analysis Software' (TAS) version 1.1 by Affymetrix. For probe analysis a bandwidth of 1 (i.e. using only data of single oligonucleotides) was applied. The signal ratio for an oligonucleotide $i$ is expressed as $D_{i}=\log _{2}\left(S_{t, i} / S_{c, i}\right)$, the $\log _{2}$ scaled quotient $D_{i}$ of probe signal $S_{t, i}$ of the treatment group and probe signal $S_{c, i}$ of the control group of arrays.

Integrated Genome Browser (IGB) was used for visualization of signal ratios.

For facilitating array analysis a flexible Java program was developed. Hereby, result text files containing probe signal and $\mathrm{p}$ values for the corresponding oligonucleotides are imported and mapped onto known genes and intergenic regions of $P$. aeruginosa PAO1. Analysis results are stored in tab-separated files including significantly detected genes. Beside obtaining gene information from an implemented file, a variable index file can be constructed from an appropriate file presented by the user. This Java program was tested on JRE 1.6.0 and is provided for download (see additional file 1).

\section{Results}

Design of a whole genome tiling array for

Pseudomonas aeruginosa PAOI

The $P$. aeruginosa Tiling Array PATA1 was designed in cooperation with and manufactured by Affymetrix according to GENECHIP ${ }^{\circledR}$ CUSTOM EXPRESS ${ }^{\text {тм }}$ ARRAY DESIGN GUIDE (Affymetrix, Santa Clara, CA). This tiling array was originally developed to screen uncharacterized $P$. aeruginosa strains for genetic differences. The genome sequence of strain PAO1 available from the Pseudomonas Genome Database (www.pseudomonas.com) was used as template for designing perfect match (PM) tiling probes with a density of $28 \mathrm{bp}(+/-5)$. The standard practice for probe selection is to prune against specific bacterial and speciesspecific controls. Pruning is a sequence comparison method and increases the quality of the unique probe selected for the design and reduces the risk of crosshybridization with other sequences (Array Design Guide, Affymetrix, Santa Clara, CA). Sequences used for hard pruning are generally highly repetitive elements, such as alu-like elements, or abundantly expressed RNA, like rRNA. Probes that cross-hybridize to hard pruning sequences were excluded. This resulted in a total of 215169 probes with 25 base pair length (Figure 1). Thus, $85.9 \%$ of the whole genome sequence $(6,264,404 \mathrm{bp})$ is present on PATA1 with an average tiling of 29 bases, hence leaving gaps of
4 bases average length (Figure 1). For two gap regions (1539 and 2907 bases) selected probes were removed as a result of hard pruning with 2 sequences - 16s rRNA and 23s rRNA (ribosomal RNAs). A second round of probe selection was started including only these gap regions and having gaps greater than $56 \mathrm{bp}$ to maximize the coverage of the tiling array while minimizing unspecific hybridization effects. By this we increased the number of probes with 204 additional probes. As negative controls we used 2358 Affymetrix Arabidopsis tiling probes as an estimate of background activity.

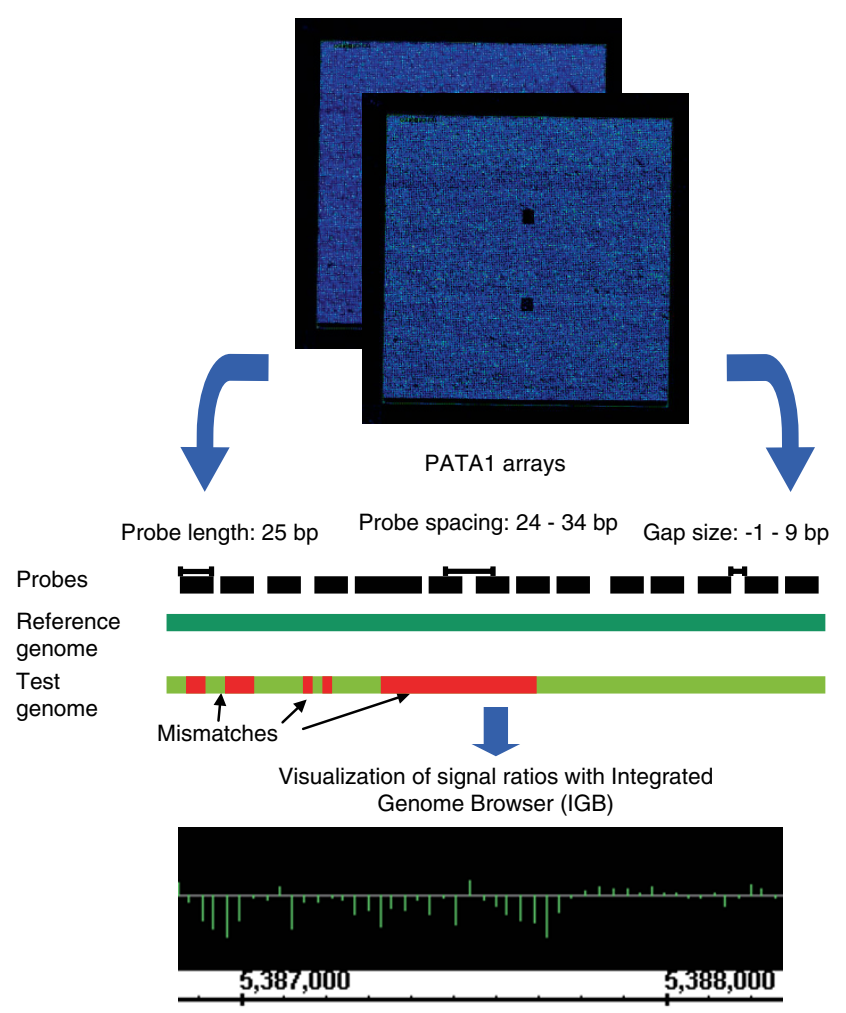

Figure I

Array design and comparative hybridization principle. The Pseudomonas aeruginosa Tiling Array (PATAI) includes 2I5000 DNA oligonucleotide probes of 25 bp (black bars). Placed with variable probe spacing (24 - 34 bp) these probes cover $85.9 \%$ of the PAOI genome leaving gaps of up to 9 bp (average $\sim 4$ ). For comparative hybridization, labeled DNA fragments of a reference strain (dark green) and the test strain (light green) were hybridized in parallel to separate PATAI arrays. Signal ratios were calculated for each oligonucleotide as $\log _{2}$ ratio of test versus reference signal and can e.g. be visualized by the Integrated Genome Browser (IGB). Genetic variations lead to mismatches of sample and probe DNA and hence to a decrease in signal ratio. 


\section{In silico comparison of PAOI array probes with the PAI4 genome sequence}

In order to estimate how many genetic differences between the two fully sequenced $P$. aeruginosa strains PAO1 and PA14 can theoretically be detected with the array we first identified in an in silico approach the best matching sequence of the published PA14 genome for each 25 bp oligonucleotide present on the array. Perfect matches (i.e. 100 per cent sequence identity) of oligonucleotides with the PA14 sequence were identified in an initial run using NCBI's BLAST software [13]. The BLAST search was sufficient for the detection of identical sequences. However, since BLAST uses a heuristic approach that approximates the Smith-Waterman algorithm [14] the alignment is fast but less accurate in finding optimal matches of non-identical sequences. Therefore all oligonucleotides for which BLAST found no perfect match were aligned in a second run using 'JAligner' [15]. JAligner is a freely available JAVA tool that makes use of the original Smith-Waterman algorithm. This algorithm is very slow (analysis of $\sim 48000$ oligonucleotides took $>1$ week computation time on a standard computer) but always finds the single optimum alignment. Combining the results from both alignments, we could determine the position of the best matching sequence within the PA14 genome, the sequence similarity, i.e. the fraction of nucleotides that is identical in both sequences that are compared, and furthermore the exact positions of mismatches and gaps. Additional to the best matching sequence, BLAST (unlike Jaligner) identified other less than optimal sequences. However, these secondary alignment hits were of much lower quality (i.e. BLAST score) and were therefore not considered for further analysis.

The sequence alignment results were subsequently used to classify the oligonucleotides according to sequence similarity (Figure 2). 166985 (77.6\%) of all oligonucleotides were found to be identical in sequence to their best matching PA14 sequence (similarity class 'identical'); 31585 (14.7\%) showed a single nucleotide polymorphism ('SNP'); 9816 (4.6\%) showed more than a single mismatch but still had at least $80 \%$ identical nucleotides with not more than 2 gaps ('partial'); and 6783 (3.2\%) had a similarity below this threshold and were therefore classified as low matching or 'absent'. It should be noted that, due to the algorithm that was applied, even absent sequences will always result in some match with low similarity that can usually be considered as random.

The core genomes of PA14 and PAO1 show a high synteny (i.e. orthologous genes are located in the same order in both genomes) with the exception of a large genomic inversion between two of the four ribosomal gene clusters $[16,17]$. This inversion is also reflected in

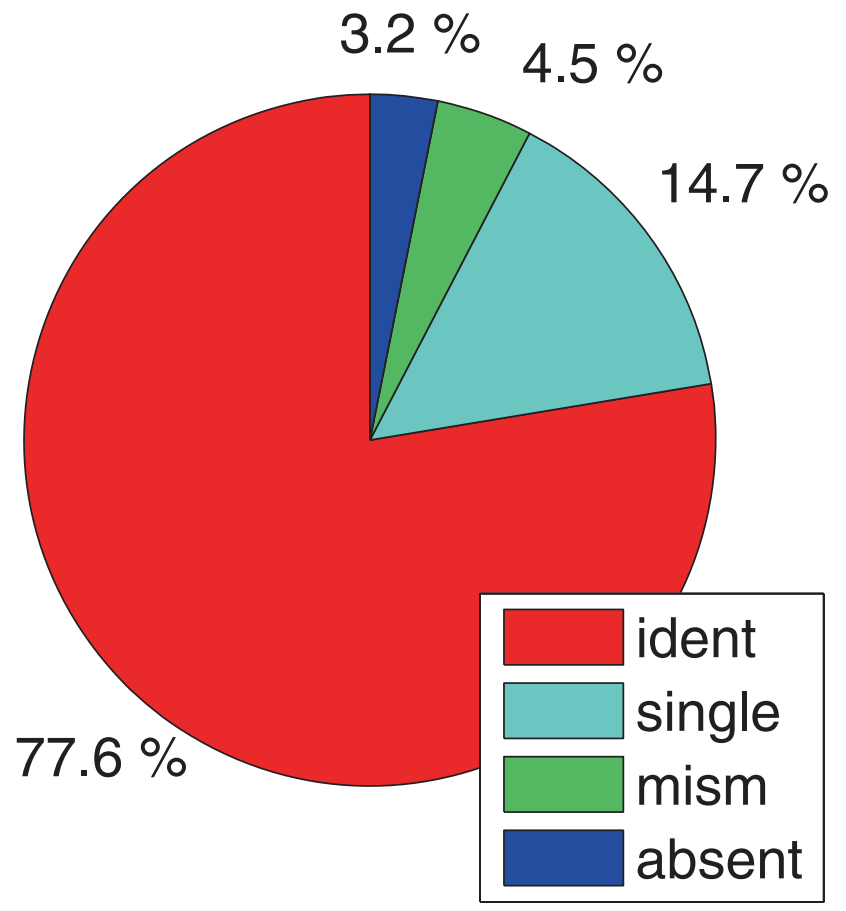

\section{Figure 2}

similarity classes resulting from global alignment of PATAI oligonucleotides with the PAI 4 genome. All oligonucleotides present on PATAI were classified by their similarity to the best matching hit resulting from an sequence alignment with the PAI4 genome sequence. Similarity classes: 'ident' - identical sequence in PAOI and PAI4, 'SNP' - single nucleotide polymorphism (SNPs), 'mism' oligonucleotides with more than a single mismatch but at least $80 \%$ sequence identity and with no more than 2 gaps, 'absent' - less than $80 \%$ sequence identity or more than 2 gaps.

the alignment data of the PATA1 oligonucleotides and the PA14 genome: for a major part of the genome the best matching sequences in PA14 were found in reversed order and on the opposite strand. The best matches of 'absent' oligonucleotides where distributed over the whole genome of PA14, which indicates that these hits are most probably random hits.

In Figure 3A we depicted a 'chromosomal map' of the PAO1 chromosome displayed as horizontal bands each one representing a region ('window') of $1 \mathrm{~kb}$ length. Regions of low sequence similarity in comparison to the PA14 genome, i.e. regions specific to PAO1, appear as darker bands (red or black) in the maps, whereas regions of high average sequence similarity are white. We furthermore compared this chromosomal map with that generated on the basis of a previously published work [16] where the authors identified 75 PAO1 specific 

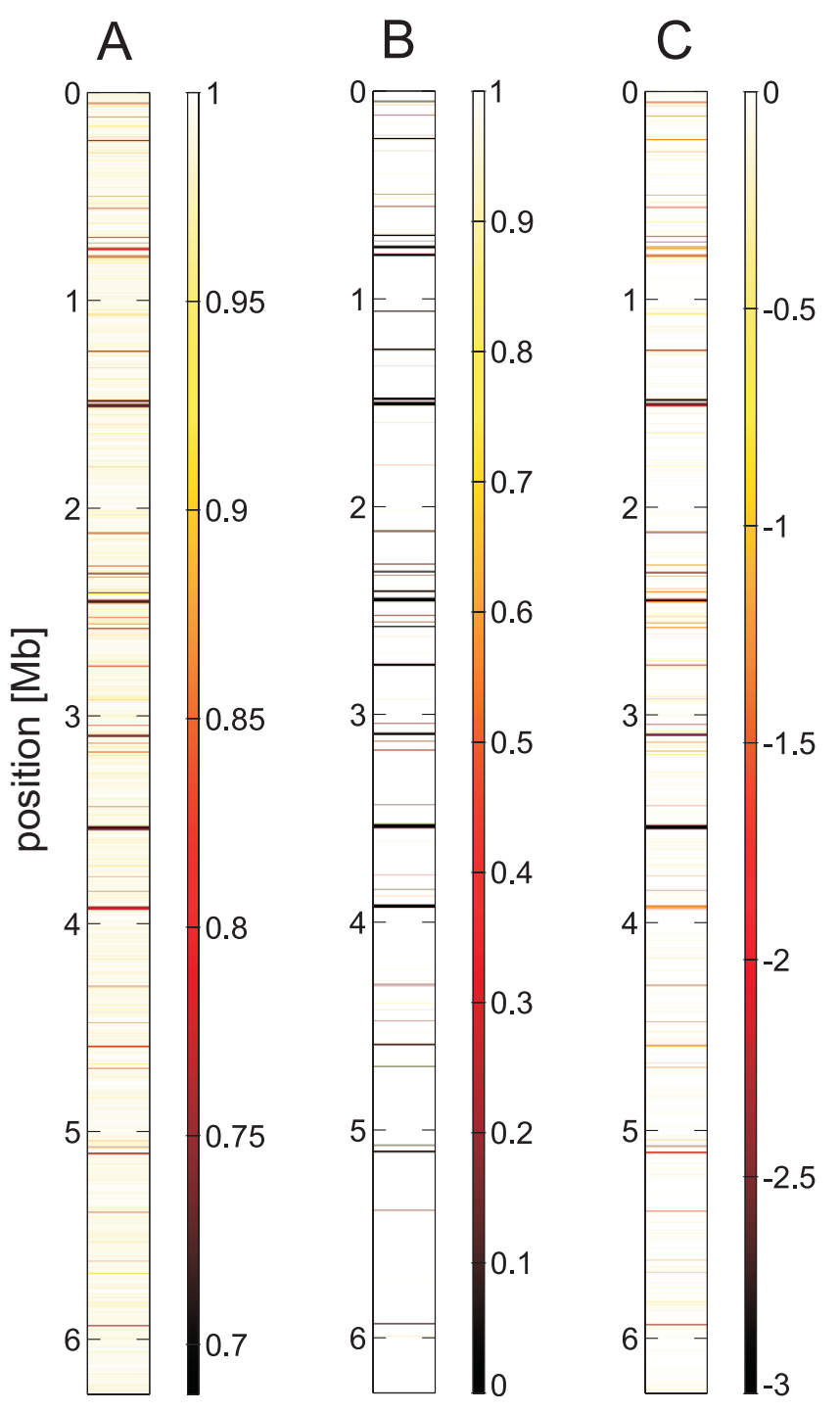

Figure 3

identification of PAOI specific regions. These 'chromosomal maps' display the whole PAOI genome as a vertical strip with horizontal bands each representing a region of $\mathrm{I} \mathrm{kb}$ at the indicated position on the chromosome with a color coded value (see color bars to the right of each map). A) Sequence similarity of PATAI oligonucleotides and the PAI 4 genome. Values indicate the fraction of nucleotides that were identical in the probe oligonucleotides and the best matching alignment in PAI4. B) PAOI specific regions. The actual value indicates the fraction of DNA which is common to PAOI and PAI4 as identified in a previous ORF-by-ORF alignment [16]. C) Signal ratio ( $\left.\log _{2}\right)$ of a comparative hybridization of PAI 4 DNA relative to PAOI DNA, calculated from data of single arrays for PAI 4 and PAOI. Values exceeding the presented interval ( -3 to 0 ) were cut off to keep the color scale in the same range as in A) and B). regions that were larger than 10 bp by an ORF by ORF alignment of the PAO1 and PA14 sequence (Figure 3B). Both analyses generate a very similar pattern suggesting that this pattern could function as a unique 'genomic fingerprint' for strain PA14. Recently, the genome-wide comparison of five P. aeruginosa strains identified regions showing significant inter-strain variation [18]. 23 regions of genomic plasticity (RGP) containing variations or insertions that are specific to PAO1 as compared to PA14 were identified and all of them are also represented in our genomic fingerprint (not shown).

\section{Comparative hybridization of the PAOI and PAI4 genomes}

To analyze the potential of PATA1 to detect genetic differences, we comparatively hybridized DNA of PAO1 and PA14 on the array. The probe signals (measured as absolute signal intensities for each oligonucleotide probe) of different oligonucleotides on a single array showed large variation ( $\sim$ by a factor of 10$)$ for both the PA14 and the reference PAO1 DNA, presumably due to different binding affinities that depend more or less directly on the oligonucleotide sequence. However, a comparison of two independent PAO1 arrays demonstrated that the variation within one microarray was reproducible and variation between data for the same probe on different arrays was considerably low (Figure 4A). A pair wise comparison of 6 independent PAO1 arrays showed high correlation of corresponding probe signals with an average correlation coefficient of $\mathrm{R}$ $=0.960$ (for 6 PA14 arrays: $\mathrm{R}=0.966$ ). When hybridizing PA14 chromosomal DNA to the array, we observed a large number of decreased probe signals putatively indicating genetic variations (Figure 4B). The signal ratios ( $\log _{2}$ scale ratios of PA14 and PAO1 probe signals) revealed the same unique pattern of PAO1 specific regions, that was already identified in the in silico sequence alignment (Figure 3C). Again, PAO1 strain specific regions appeared as darker bands on a bright background of regions with high sequence similarity. The highly reproducible fingerprint like pattern of sequence similarity on the chromosomal scale does not only provide a tool for strain identification, but also allows detailed determination of the genomic structure, e.g. presence and location of genomic islands and strain specific regions.

As opposed to the identification of the presence or absence of strain specific regions, the detection of inter-strain variations at the level of single oligonucleotides is less clear cut. Signal ratios of variant and unchanged regions are overlapping as can be observed in the scatter plot of the 
comparative hybridization of PAO1 and PA14 (Figure 4B). Therefore, any threshold applied to filter out variant signal ratios will lead to disregard of some variant regions because their signal ratio is not changed enough (false negatives) and on the other hand detection of unchanged regions that show for some reason a variation to a lowered signal ratio (false positives). The quality of the set of oligonucleotides that is detected as variant by applying a certain threshold can be expressed in terms of sensitivity and specificity of the detection. We defined an oligonucleotide $i$ as 'detected' (i.e. showing a reduced signal ratio that supposedly indicates a sequence variation), if its signal ratio $D_{i}$ was below $D_{t h r}$ the signal ratio threshold $\left(D_{i} \leq D_{t h}\right)$. The sensitivity of detection was defined as

$$
\operatorname{sens}\left(D_{t h}\right)=\frac{N_{\operatorname{var}}\left(D_{t h}\right)}{N_{\mathrm{var}}^{0}}
$$

where $D_{t h}$ denotes the signal ratio threshold, $N_{\mathrm{var}}\left(D_{t h}\right)$ the number of oligonucleotides with sequence variations (as identified in silico) that showed a signal ratio $D_{i} \leq D_{t h}$ (i.e. true positive detections), and $N_{\mathrm{var}}^{0}$ the total number of variant oligonucleotides. Thus, sensitivity describes
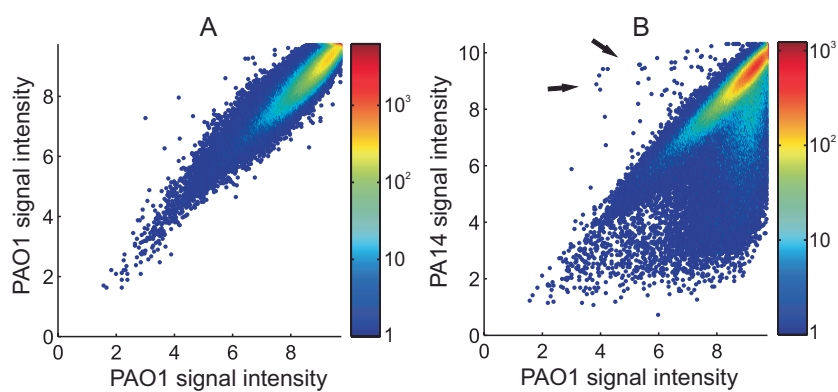

\section{Figure 4}

hybridization ratio variation. Scatterplots showing the difference in hybridization signal between two independent arrays. Data points represent normalized signal intensities ( $\log _{2}$ scale) of oligonucleotides. Colors indicate the number of data points that have been grouped for a better visualization (see color bar for scale). A) Intra strain variation of signal intensity. Two independent samples of PAOI DNA were comparatively hybridized. Signal intensities show high correlation $(R=0.935)$ with most data points being close to the diagonal. B) Variation of signal intensity between PAI4 and PAOI. Two single arrays were comparatively hybridized with PAI4 DNA and PAOI DNA, respectively. The large cloud of data points below the diagonal indicates sequence variations that lead to decreased signal ratios for the affected oligonucleotide probes. Data points significantly above the diagonal (arrows) are due to a I kb deletion in the reference PAOI strain (for details see Figure 7 and text). Note that a small proportion $(\sim 2 \%)$ of signals shows saturation effects leading to the strict edges of the data clouds. how many oligonucleotides that are different to the PA14 sequence actually are detected upon application of a certain $D_{t h}$.

Furthermore, we defined specificity as

$$
\operatorname{spec}\left(D_{t h}\right)=\frac{N_{\operatorname{var}}\left(D_{t h}\right)}{N_{\text {total }}\left(D_{t h}\right)}
$$

where $N_{\text {total }}\left(D_{t h}\right)$ is the number of all oligonucleotides with $D_{i} \leq D_{t h}$ (including false positives). Hence, specificity describes the fraction of correct detections (true positives) among all detections.

In order to find a threshold optimizing sensitivity and specificity we determined the number of detected oligonucleotides for a range of $D_{t h}$ values based on a data set obtained from comparative hybridization of 2 independent
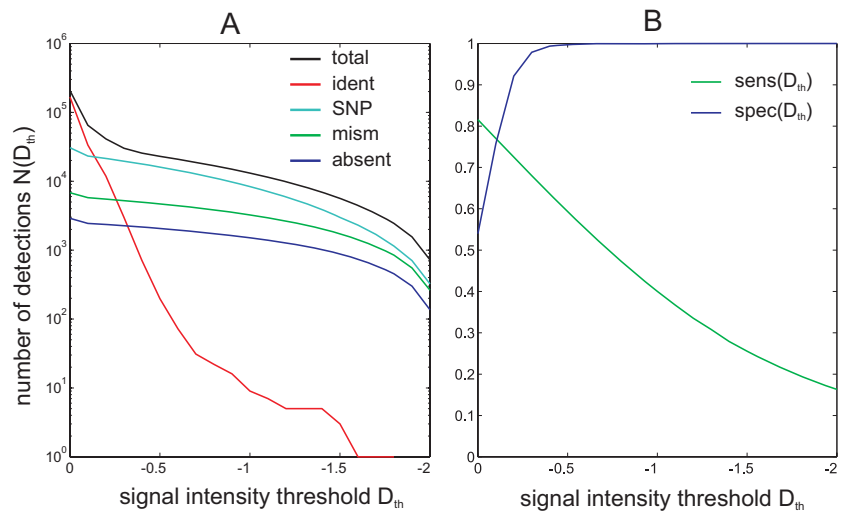

Figure 5

detection of variations - sensitivity and specificity. Detection of variant oligonucleotides from a comparative hybridization of PAI4 and PAOI DNA. For each strain two independent arrays were used. A) Detection of certain variations by similarity class (Figure 2 ) for different values of signal ratio threshold $D_{t h}$. More restrictive (i.e. more negative) threshold values reduce the number of overall detections (total) but false positive detections (ident) are affected more than the others. Each curve represents the number of oligonucleotides that are detected (i.e. that show a signal ratio $D_{i} \leq \mathbf{D}_{t h}$ ) using a given $D_{t h}$. total (black curve) indicates the sum of all detections, while ident (red), SNP (cyan), mismatch (green) and absent (blue) refer to the detection numbers of the respective similarity classes as described in Figure 2. B) Sensitivity and specificity. Sensitivity is defined as the fraction of variant oligonucleotides that are actually detected using a certain threshold $D_{t h}$ (Equation I). Specificity is defined as the ratio of false positive detections (such of 'identical' oligonucleotides) among all detections (Equation 2). The dashed vertical line indicates a threshold $D_{t h}=-0.5$ as it is used in the text. 
arrays for both PA14 and PAO1 (Figure 5). Application of a more restrictive, i.e. more negative, $D_{t h}$ generally reduced the detection, as reflected by the detection counts (Figure 5A) and thus the sensitivity (Fig 5B). E.g., more than $10^{4}$ variant oligonucleotides were detected using $D_{t h}=-1$ while only $\sim 10$ false positives remained (Figure 5A). However, this high specificity of $\sim 0.9996$ (Figure 5B) lead to a drop in sensitivity to 0.40 , meaning $60 \%$ true hits were missed in this case. A significantly higher sensitivity of 0.59 resulted for $D_{t h}=-0.5$ while specificity was still high (0.997). Sensitivity further increased with a less restrictive $D_{t h}$ but at the cost of a strong decrease in specificity (Figure 5B).

Remarkably, such high sensitivities could already be achieved by comparative hybridization of only one single array for each PAO1 and PA14, though the values showed variation when different arrays were compared (Table 1). Including more arrays in an analysis (which would normally be avoided because of the cost factor) did not increase the sensitivity. Specificity, on the other hand, increased with larger sets of arrays (Table 1) indicating that the observed variation in sensitivity is caused by falsely positive detection of oligonucleotides, which occurs randomly. When attempting to identify genetic variations in uncharacterized strains, it seems therefore reasonable to use even only one array for both the test and reference strain as a first scan for putative variations. More arrays could then be used to increase specificity as a validation of results, although this could as well be achieved by less sophisticated (and costly) methods such as sequencing of a putative variant gene.

\section{Detection of variations at the gene level}

Since the Tiling Analysis Software (TAS) provided by Affymetrix can process the results of a comparative hybridization only at the level of oligonucleotides and a user usually might be more interested in the genes that are affected by the detected variations, we also developed a Java software tool to translate these results to the level of genes. The software reads the output data produced by TAS and applies a signal threshold $D_{t h}$ to filter out candidate variant oligonucleotides. The oligonucleotide positions are then used to map these detections to the corresponding genes or intergenic regions. As an example, Table 2 shows the results of both the sequence alignment and a comparative hybridization analyzed with TAS and our JAVA tool for the gene clusters containing $w b p$ and related genes that are involved in the synthesis of lipopolysaccharides (LPS). PAO1 contains two clusters of $w b p$ genes, one of which (PA3141PA3160) is specific to PAO1 [18] while the other (PA5447-PA5452) is present in both PAO1 and PA14. In case of the specific genes of the PA3141-PA3160 cluster, most of the oligonucleotides covering those genes belonged to the 'mism' or 'absent' similarity class in the in silico alignment. The comparative hybridization also detected most of the oligonucleotides of the specific genes as absent, which is also reflected by the high sensitivity close to $100 \%$. The other gene cluster showed a high sequence similarity in both strains with a few SNPs present in each gene (Table 2). Detection of those SNPs showed sensitivities around $40-60 \%$ corresponding to the global average for SNPs.

\section{Detection of mismatches is affected by mismatch position and the type of nucleotide substitution}

The detection of mismatches by comparative hybridization relies on the decreased formation of probe/sample DNA duplexes. It has been show previously that the hybridization of DNA fragments on surface tethered probes is affected by various factors, such as the position of the mismatch within the duplex and the stacking energy at this position [19], or surface properties of the array [20]. In our in silico analysis 31585 oligonucleotides with single nucleotides polymorphisms (SNPs) could be identified ('SNP', Figure 2), enabling a detailed analysis of SNP detection from our experimental data. Grouping all SNP oligonucleotides according to the location of the mismatch

Table I: Influence of the size of data sets on the sensitivity and specificity of different data sets

\begin{tabular}{|c|c|c|c|c|c|c|}
\hline \multirow[b]{2}{*}{$\begin{array}{l}\text { treatment group } \\
\text { size }^{\mathrm{a}}\end{array}$} & \multirow[b]{2}{*}{ control group size ${ }^{\mathrm{a}}$} & \multirow[b]{2}{*}{ total } & \multicolumn{2}{|c|}{ sensitivity } & \multicolumn{2}{|c|}{ specificity } \\
\hline & & & SNP & mismatch & absent & total \\
\hline I & 1 & 0.585 & $0.48 I$ & 0.740 & 0.848 & 0.989 \\
\hline 1 & 1 & 0.600 & 0.500 & 0.747 & 0.860 & 0.967 \\
\hline I & 1 & 0.588 & 0.479 & $0.75 I$ & 0.866 & 0.998 \\
\hline I & 1 & 0.603 & 0.497 & 0.759 & 0.874 & 0.989 \\
\hline 2 & 2 & 0.593 & 0.488 & 0.750 & 0.863 & 0.997 \\
\hline 4 & 4 & 0.590 & 0.483 & 0.752 & 0.866 & 0.998 \\
\hline 6 & 6 & 0.572 & 0.459 & 0.742 & 0.863 & 0.999 \\
\hline
\end{tabular}

${ }^{\text {a }}$ The four analyses of $2 \times 1$ array are the four possible combinations of arrays that were used in the $2 \times 2$ analysis. The $2 \times 4$ and $2 \times 6$ analyses contain the above stated microarrays plus additional ones. 
Table 2: In silico comparison and comparative hybridization results for the two wbp gene clusters of PAOI

\begin{tabular}{|c|c|c|c|c|c|c|c|c|c|c|}
\hline \multicolumn{3}{|c|}{ gene information } & \multicolumn{6}{|c|}{ in silico analysis } & \multicolumn{2}{|c|}{$\begin{array}{l}\text { comparative } \\
\text { hybridization }\end{array}$} \\
\hline \multirow[b]{2}{*}{$\begin{array}{l}\text { PAOI } \\
\text { locus ID }\end{array}$} & \multirow[b]{2}{*}{ PAI4 locus ID } & \multirow[b]{2}{*}{ gene name } & \multicolumn{5}{|c|}{ count of similarity classes ${ }^{\mathrm{a}}$} & \multirow[b]{2}{*}{$\begin{array}{c}\text { average } \\
\text { sequence } \\
\text { similarity }\end{array}$} & \multirow[b]{2}{*}{$\begin{array}{l}\text { detected } \\
\text { oligos }^{\mathrm{b}}\end{array}$} & \multirow[b]{2}{*}{ sensitivity $^{\mathrm{b}}$} \\
\hline & & & ident. & SNP & mism. & absent & total & & & \\
\hline PA3|4I & PAI4_23470 & $w b p M$ & 54 & 7 & 4 & 5 & 70 & 0.97 & 12 & 0.75 \\
\hline PA3|42* & absent & & 4 & 3 & 4 & I & 12 & 0.93 & 7 & 0.88 \\
\hline PA3।43* & absent & & 4 & 4 & 8 & 3 & 19 & 0.90 & II & 0.73 \\
\hline РА3।44* & absent & & I & 0 & 4 & 0 & 5 & 0.91 & 4 & 1.00 \\
\hline РA3।45* & PAI4_23460 & wbpl & 0 & 0 & 3 & 33 & 36 & 0.71 & 35 & 0.97 \\
\hline РА3।46* & PAI4_23450 & wbpK & 0 & 0 & 6 & 28 & 34 & 0.71 & 33 & 0.97 \\
\hline PA3 I 47* & absent & $w b p J$ & 0 & 0 & 6 & 37 & 43 & 0.72 & 43 & 1.00 \\
\hline РА3।48* & absent & wbpl & 0 & 0 & 7 & 30 & 37 & 0.74 & 37 & 1.00 \\
\hline PA3।49* & absent & $w b p H$ & 0 & 0 & 6 & 33 & 39 & 0.72 & 39 & 1.00 \\
\hline PA3।50* & absent & wbpG & 0 & 0 & 3 & 36 & 39 & 0.70 & 39 & 1.00 \\
\hline PA3I5I* & absent & hisF2 & 0 & 0 & 3 & 24 & 27 & 0.69 & 26 & 0.96 \\
\hline РA3152* & absent & his $\mathrm{H}_{2}$ & 0 & 0 & I & 21 & 22 & 0.70 & 22 & 1.00 \\
\hline PA3।53* & absent & wzx & 0 & 0 & 1 & 43 & 44 & 0.71 & 43 & 0.98 \\
\hline РA3।54* & absent & wzy & 0 & 0 & I & 45 & 46 & 0.70 & 46 & 1.00 \\
\hline РA3।55* & absent & wbpE & 0 & 0 & 5 & 33 & 38 & 0.72 & 38 & 1.00 \\
\hline PA3I56* & absent & wbpD & 0 & 0 & 4 & 17 & 21 & 0.72 & 20 & 0.95 \\
\hline РА3।57* & absent & & 0 & 0 & 14 & 51 & 65 & 0.73 & 64 & 0.98 \\
\hline РА3।58* & absent & $w b p B$ & 0 & 0 & 7 & 26 & 33 & 0.74 & 33 & 1.00 \\
\hline РА3।59* & absent & $w b p A$ & 0 & 0 & 14 & 32 & 46 & 0.73 & 46 & 1.00 \\
\hline PA3। 60 & PAI4_23360 & wzz & 0 & 0 & 5 & 31 & 36 & 0.71 & 36 & 1.00 \\
\hline PA3I6I & PAI4_23340 & $\operatorname{himD}$ & 8 & 1 & 1 & 0 & 10 & 0.99 & I & 0.50 \\
\hline PA5447 & PAI4_7I9I0 & $w b p Z$ & 35 & 4 & I & 0 & 40 & 0.99 & 5 & 1.00 \\
\hline PA5448 & PAI4_7I920 & $w b p Y$ & 34 & 5 & 0 & 0 & 39 & 0.99 & 2 & 0.40 \\
\hline PA5449 & PAI4_7I930 & $w b p X$ & 40 & 8 & 1 & 0 & 49 & 0.99 & 4 & 0.44 \\
\hline PA5450 & PAI4_7|940 & wzt & 40 & 5 & 0 & 0 & 45 & 1.00 & 3 & 0.60 \\
\hline PA545 I & PAI4_7I960 & wzm & 24 & 5 & 0 & 0 & 29 & 0.99 & 3 & 0.60 \\
\hline PA5452 & PAI4_7I970 & wbpW & 42 & 6 & I & 0 & 49 & 0.99 & 4 & 0.57 \\
\hline
\end{tabular}

* genes belonging to the PAOI specific region.

a see Figure 2 for a definition of the similarity classes.

bdetermined for $D_{t h}=-0.5$.

demonstrated that mismatches at marginal positions mostly lead to a much smaller decrease of signal ratio as compared to mismatches located at a more central position (Figure 6A). The average signal ratio for a mismatch at a central position was about -0.7 and approached zero at the positions close to either of both ends, which also severely affected the sensitivity (Figure 6B). This significant drop in sensitivity can be observed at the 4 outermost positions of either side $(1-4,22-25)$, while sensitivity was relatively similar (around 60\%) for the central 17 positions.

To analyze the influence of the type of nucleotide present in the mismatching position we grouped all SNPs according to the alleles present in the PATA1 probes and the PA14 sequence, respectively. This analysis revealed a marked bias in the median (average) signal ratios of certain SNPs (Table 3 ). The median signal ratios differed from -0.57 for SNPs where a 'T' within the probe corresponded with a ' $\mathrm{C}$ ' within the PA14 sequence
(PAO1-T/PA14-C) down to only -0.24 for SNPs with alleles PAO1-C/PA14-A. Most notably, this effect seemed to be asymmetric, since the median signal ratios for opposite SNPs, like e.g. PAO1-T/PA14-A and PAO1-A/ PAO1-T, showed large differences in most cases (Table 3).

Furthermore, grouping the different SNPs according to the category of the nucleotide substitution revealed that the bias observed in our data strongly depended on the 'interaction type' of the nucleotides present in either allele. The nucleotides $\mathrm{G}$ and $\mathrm{C}$ can be categorized as showing 'strong interaction' (common symbol in IUPAC format: 'S') because they can form three hydrogen bonds in contrast to $\mathrm{A}$ and $\mathrm{T}$ which show only weak interaction ('W'). While any SNP constitutes a mispairing and thus no regular $\mathrm{H}$ bonds are formed, the median signal ratio was different for different combinations of interaction types (S or W) in the probe and sample alleles (Table 4). 

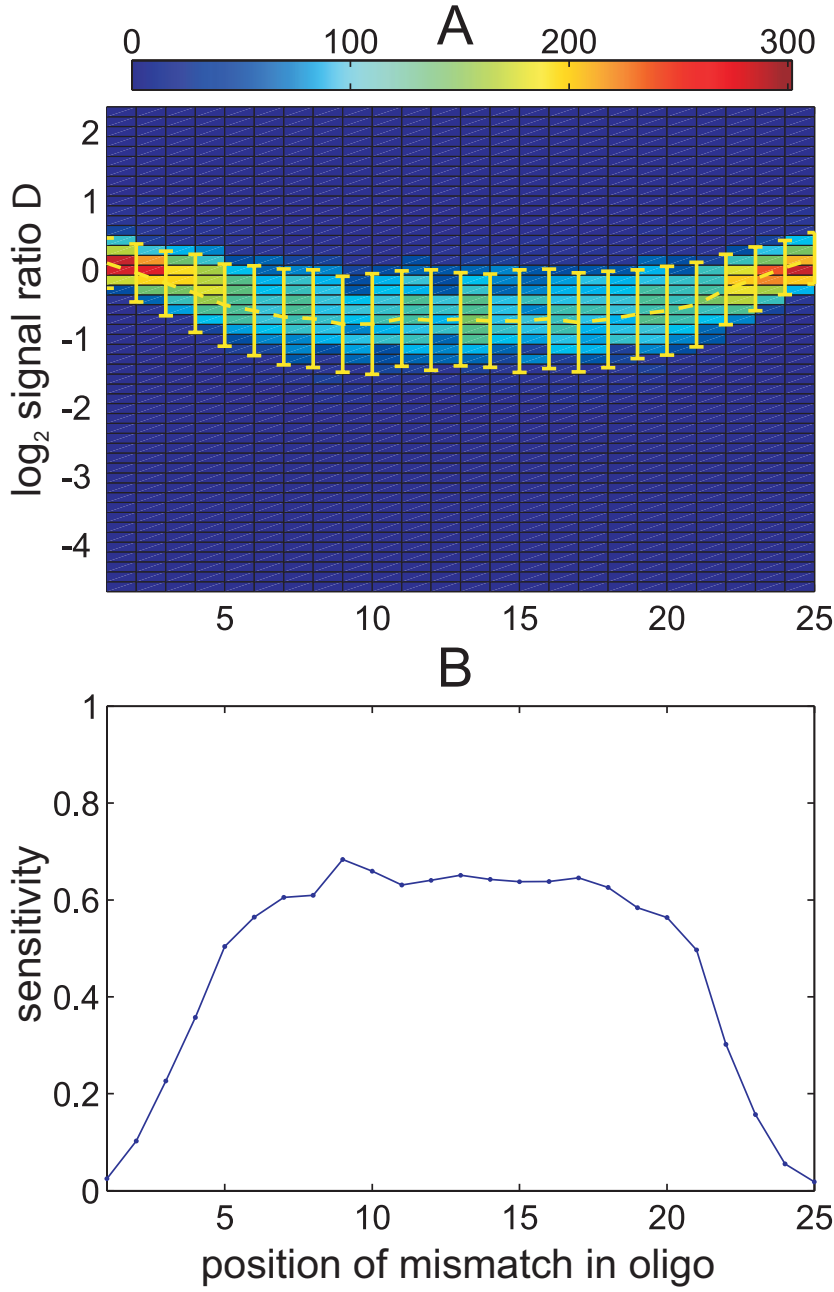

Figure 6

mismatches placed at the margins of oligonucleotides are hard to detect. A) 3D histogram grouping oligonucleotides according to mismatch location and signal ratio ( $\log _{2}$ scaled). Only oligonucleotides covering a single nucleotide polymorphism (SNP) with the corresponding PAI4 sequence are included. Each rectangle represents the group of all oligonucleotides with a SNP at the specified position ( $x$-axis) showing the specified hybridization ratio ( $y$-axis). The dashed yellow line indicates the average hybridization ratio of all oligonucleotides with common mismatch location. Error bars indicate one standard deviation. B) Sensitivity (see Figure 5) for detection of oligonucleotides covering a SNP $\left(D_{t h}=-0.5\right)$.

The strongest decrease in signal ratio (-0.53) was observed for SNPs, where an S nucleotide in the PA14 sample DNA corresponded to $\mathrm{W}$ in the PAO1 probe (PAO1-W/PA14-S). On the other hand, SNPs with PAO1-S/PA14-W showed only a median signal ratio of -0.30. This asymmetry cannot be explained by the
Table 3: Median signal ratio of SNPs grouped by the bases present in both alleles.

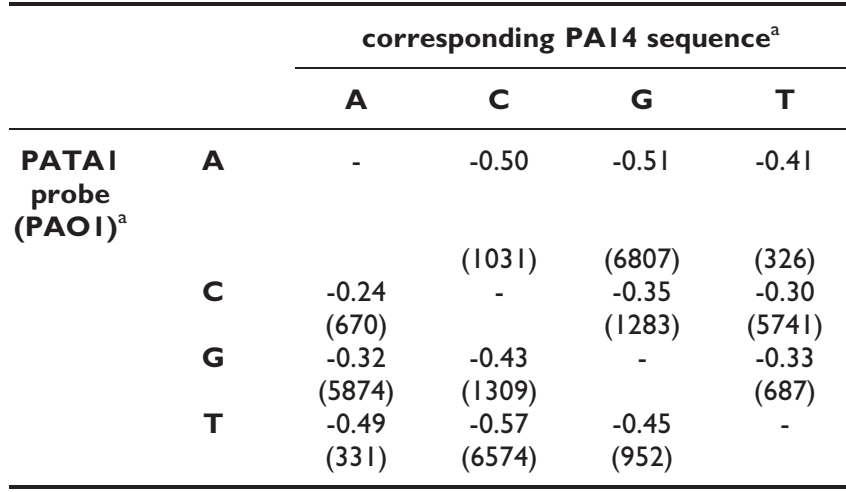

Numbers in brackets indicate the abundance of each SNP in our data. a mismatching nucleotide in the oligonucleotide probe and in the PAI4 sequence, respectively.

sequence, which is the same except for the mismatch itself, and probably results from the surface fixation of the probe. It has been shown previously that surface effects affect duplex formation [19, 20].

The marked differences in abundance of SNP types (Table 3) result mostly from a biased transition/transversion ratio. According to our data, transitional substitutions, i.e. mutations from purine $(\mathrm{G}, \mathrm{A})$ to purine or pyrimidine $(\mathrm{C}, \mathrm{T})$ to pyrimidine, occur about 4 times as often as transversions (purine to pyrimidine or vice versa) (Table 5). However, the signal ratios of transversions and transitions did not show any significant bias.

We also examined the influence on the signal ratio of the nucleotides that are direct neighbors of the mismatch. There are 64 possible trinucleotides formed by the SNP nucleotide and its direct neighbors and 3 different alleles forming a mismatch at the central position with these trinucleotides. The results for each of these 192 different combinations is provided in additional file 2 . The signal ratio is on average less negative if a SNP is flanked by strongly interacting bases ( $\mathrm{G}$ or $\mathrm{C}$ ) as summarized in Table 6. Therefore, such SNPs are more difficult to detect as indicated by the lower sensitivity.

\section{Detection of a l $\mathbf{k b}$ deletion in the Washington Genome Center PAOI strain}

Since PA14 is closely related to PAO1 and more than $75 \%$ of the PATA1 oligonucleotides correspond to identical sequences in PA14, the comparative hybridization of PAO1 and PA14 DNA should allow not only the detection of variations in PA14 as opposed to PAO1 but also of variations in the chromosomal DNA of PAO1 as opposed to the hypothetical PAO1 sequence which was 
Table 4: Signal ratios of SNPs are biased towards the interaction type (strong or weak) of the bases present in the probe and sample alleles.

\begin{tabular}{ccccc}
\hline PAOI allele (probe) $^{\mathrm{a}}$ & PAI4 allele (sample) $^{\mathrm{a}}$ & abundance & median signal ratio $^{\text {sensitivity }^{\mathrm{b}}}$ \\
\hline W & $\mathrm{S}$ & 15364 & -0.53 & $52.1 \%$ \\
W & W & 657 & -0.46 & $47.5 \%$ \\
S & S & 2592 & -0.38 & $43.7 \%$ \\
S & W & 12972 & -0.30 & $39.1 \%$ \\
\hline
\end{tabular}

${ }^{a}$ W - IUPAC format for bases with weak interaction (A, T), S - strong interaction (G, C).

${ }^{\mathrm{b}}$ determined for $D_{t h}=-0.5$.

Table 5: Signal ratios of SNPs are independent from the chemical class (pyrimidine or purine) of the bases present in the probe and sample alleles.

\begin{tabular}{|c|c|c|c|c|c|}
\hline & PAOI allele (probe) ${ }^{a}$ & PAI 4 allele (sample) ${ }^{a}$ & abundance & median signal ratio & sensitivity $^{\mathrm{b}}$ \\
\hline \multirow[t]{2}{*}{ transitions } & $Y$ & $Y$ & 12315 & -0.44 & $46.9 \%$ \\
\hline & $\mathrm{R}$ & $\mathrm{R}$ & $|268|$ & -0.43 & $45.8 \%$ \\
\hline \multirow[t]{3}{*}{ transversions } & $\mathrm{R}$ & $Y$ & 3353 & -0.44 & $46.9 \%$ \\
\hline & Y & $\mathrm{R}$ & 3236 & -0.37 & $43.0 \%$ \\
\hline & & & 31585 & -0.43 & $45.9 \%$ \\
\hline
\end{tabular}

${ }^{a} Y$ - IUPAC format for pyrimidine bases $(C, T), R$ - purine bases $(A, G)$.

b determined for $D_{t h}=-0.5$.

Table 6: Signal ratios of SNPs are influenced by interaction of neighboring nucleotides

\begin{tabular}{cccc}
\hline $\begin{array}{c}\text { neighboring } \\
\text { nucleotides }\end{array}$ & abundance & $\begin{array}{c}\text { median signal } \\
\text { ratio }\end{array}$ & sensitivity $^{\mathbf{b}}$ \\
\hline W-W & 3223 & -0.77 & $63.2 \%$ \\
$W^{\mathrm{a}}$-S or S-W & 14599 & -0.54 & $52.5 \%$ \\
S-S & 11123 & -0.39 & $42.7 \%$ \\
marginal $^{c}$ & 2640 & 0.07 & $2.2 \%$ \\
\hline & 31585 & -0.43 & $45.9 \%$ \\
\hline
\end{tabular}

${ }^{a}$ W - IUPAC format bases A, T showing weak interaction, $S$ - IUPAC for $\mathrm{G}, \mathrm{C}$ showing strong interaction.

b determined for $D_{t h}=-0.5$.

c SNPs in the marginal position I or 25 that don't have two neighboring nucleotides (see also Figure 6).

used for designing the PATA1 oligonucleotide probes. In contrast to the above described variations in PA14, those PAO1 variations should appear as an increase in probe signal. Indeed, we identified a whole set of 35 oligonucleotides with signal ratios between $0.97(\sim 2$ fold increase) and 5.17 ( $\sim 36$ fold increase), which all together covered parts of the two hypothetical ORFs PA4684 and PA4685. Since the increased signal ratio resulted from a decrease in the PAO1 probe signals while PA14 probe signals were unaffected, we postulated a partial deletion of these two genes in the Washington
PAO1 strain to be the cause of the observation. This hypothesis was tested by PCR amplification of (i) the whole region including short parts of the flanking sequence (primers A1, A2) and (ii) a shorter sequence inside the hypothetical deletion (primers B1, B2). The size of this deletion could be predicted to be between 1005 - 1020 bp considering the location to the unaffected oligonucleotide that were closest to that region. The PCR results using PAO1 and PA14 chromosomal DNA, respectively, clearly indicated a size shift of the expected band for primer pair A1/A2 of 1000 bp which perfectly fits the prediction (Figure 7) and with primers B1/B2 no PCR product was observed for PAO1. As an alternative to the Washington Genome Center PAO1 strain that was used for microarray hybridization, we also tested PAO1 DSM1707 which like PA14 resulted in PCR products corresponding to the published sequence of PA4684/4685. These results clearly indicate a genetic difference between different strains of PAO1.

\section{Discussion}

In this study we have successfully designed and evaluated a tiling microarray which targets the whole chromosome of the facultative pathogen $P$. aeruginosa PAO1. Given PAO1's large genome size and a limited construction capacity, one microarray depicted the genome with 25-base-long oligonucleotides tiled with an average 29 base pair spacing 


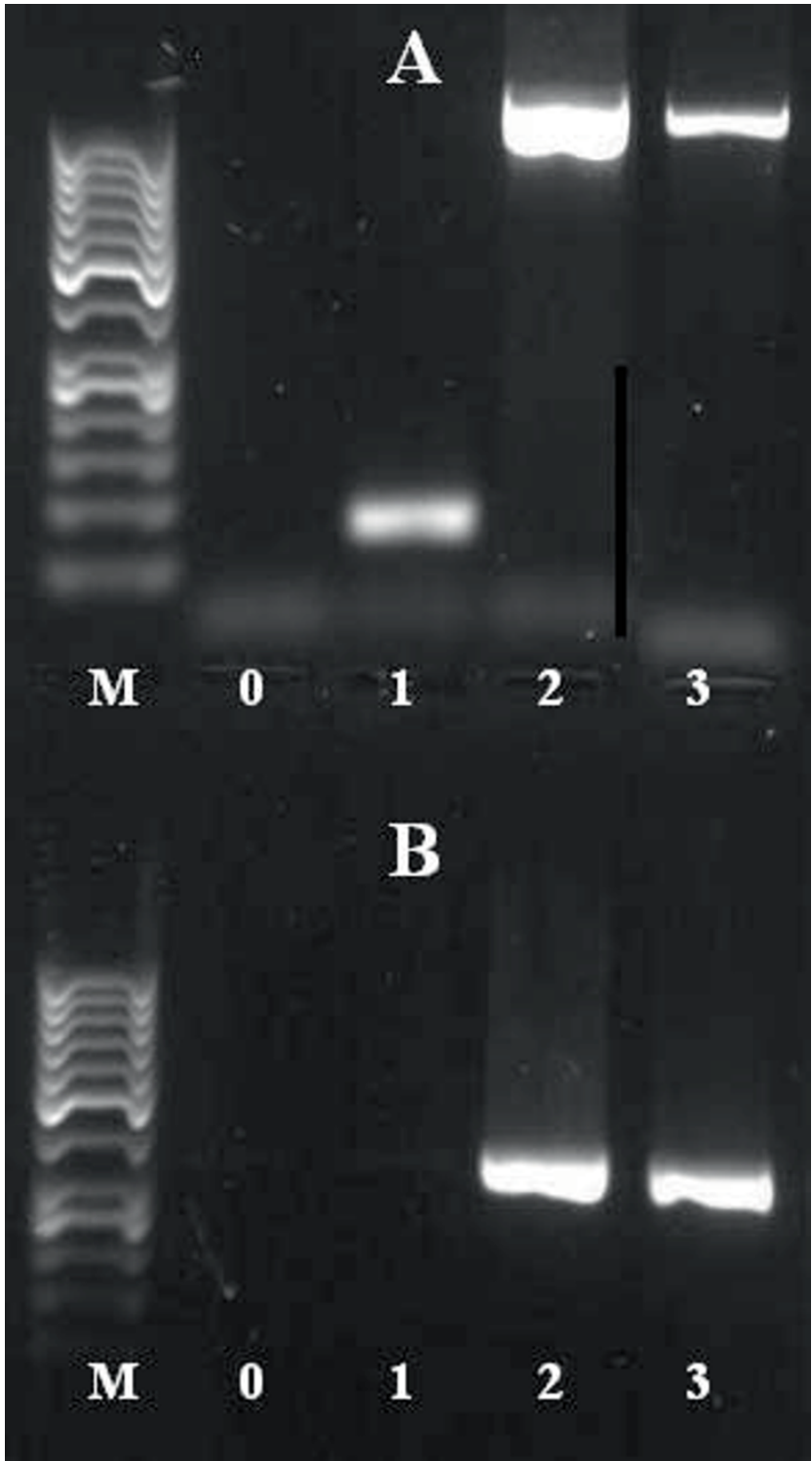

Figure 7

Identification of a I kb deletion in MPAO I.

Gelelectrophoresis after PCR Amplification of the PA4685 region using primer pair AI/A2 ('A') or BI/B2 ('B').

$M$-GeneRuler 50 bp (Fermentas, Hanover, MD), 0 - control PCR lacking chromosomal DNA, I - PAOI Washington Genome Center, 2 - PAI4, 3 - PAOI DSMI707.

which corresponded to an average gap between the oligonucleotides of 4 base pairs.

The alignment of all PAO1 derived 25 base pair sequences present on the $P$. aeruginosa microarray with the published genome sequence of strain PA14 identified regions specific to PAO1 that largely correspond the results of previous studies based on ORF-by-ORF alignment [16]. Experimental data from comparative hybridization of PA14 and PAO1 DNA on the PATA1 chip reproduced this pattern at the chromosomal scale (Figure 3), indicating that genetic differences affecting multiple consecutive oligonucleotides on the array can be detected at very high sensitivity and specificity, even when data were obtained from only one comparative hybridization.

The comparative hybridization of a reference PAO1 obtained from the Washington Genome Centre and the PA14 strain, furthermore lead to the identification of a 1 $\mathrm{kb}$ deletion in the Washington PAO1 strain, as compared to the published PAO1 sequence. This deletion affected part of the PA4684 and almost the whole PA4685 gene and explains why in the Washington transposon mutant collection no insertions within the PA4685 gene have been found.

Whereas genetic differences affecting multiple consecutive oligonucleotides on the array can easily be detected, we were also interested in the performance of the $P$. aeruginosa genome array in the identification of single-base pair changes. A similar microarray has previously been developed and successfully applied for the identification of even single-point mutations leading to metronidazole resistance in $H$. pylori strains [11]. To evaluate the performance of the PATA1 array we compared an in silico alignment with experimental comparative hybridization data in great detail and identified three factors influencing the sensitivity in the detection of single nucleotide mismatches: i) positional bias - mismatches located at the 4 marginal positions from either side of the oligonucleotide were detected with a much lower sensitivity than mismatches at central locations, leaving a core region of 17 nucleotides; ii) interaction sites bias - sensitivity was decreased for mismatches where a $\mathrm{C}$ or $\mathrm{G}$ ( 3 possible $\mathrm{H}$ bonds) on the $\mathrm{PAO} 1$ probe sequence was corresponding to an $\mathrm{A}$ or $\mathrm{T}$ (2 possible $\mathrm{H}$ bonds) in the PA14 sample sequence and vice versa; and iii) neighbor bias - sensitivity was increased if the nucleotides neighboring the mismatch showed weaker binding, i.e. fewer $\mathrm{H}$ bonds (A or T), and vice versa.

Because the sensitivity towards point mutations was significantly reduced at the outer 4 positions at either end of an oligonucleotide, the core regions of all oligonucleotides covered only $\sim 58 \%$ of the PAO1 chromosome effectively. This limitation leaves space for a next microarray generation which should depict the whole genome with 25-base-long oligonucleotides tiled with a 16 base pair or less spacing (corresponding to more than 400.000 oligonucleotides). However, despite this low effective coverage, about $50 \%$ of all single nucleotide polymorphisms (SNPs) theoretically covered 
by the PATA1 array could be detected in a comparative hybridization of PAO1 and PA14 genomes using a threshold hybridization ratio of $D_{t h}=-0.5$. Variations larger than SNPs were detected with a much higher sensitivity (up to $85 \%$ ) leading to an overall detection of about $60 \%$ of all theoretical variations with high specificity.

Our results clearly indicate that the microarray hybridization presented in this study represents a very robust method to screen whole sets of $P$. aeruginosa strains bearing unknown genetic variations. One attractive future application of these microarrays could be to identify and depict the $P$. aeruginosa genome organization of clinical strains. Most of the CF patients acquire $P$. aeruginosa from the environment early in life and suffer from transient airway infections with diverse strains of $P$. aeruginosa, whereas at a later stage the patients become permanently colonized with one or few P. aeruginosa clones [8]. Although diverse environmental $P$. aeruginosa isolates cause chronic infections in the CF lung, it has recently been reported that there are dominant clones in the environment and disease and that individual clones prefer a specific repertoire of accessory segments [21]. In contrast to the core genome, which is mostly shared by all $P$. aeruginosa strains, the accessory genome is highly strain specific [18].

The microarray could be used as a highly sophisticated fingerprinting method to significantly advance the question of whether there are specific genome organizations or certain genetic elements in clinical strains that are more frequently associated with chronic disease and with adverse clinical outcome. These traits may serve as important prognostic markers and may be targets of future drugs designed specifically for action against chronic infections. The recent development of next generation microarrays will offer the opportunity to include strain specific markers (pathogenicity islands) of common $P$. aeruginosa clones.

Furthermore arrays that cover the whole $P$. aeruginosa genome (with tighter tiling) might serve as an alternative cost-effective method and a clear alternative to whole genome sequencing strategies for the identification of genetic variations. The availability of an easy to perform mutation discovery method in $P$. aeruginosa will make a very important contribution and will significantly advance the field of patho-adaptive $P$. aeruginosa evolution during the chronic infection process. $P$. aeruginosa undergoes an intense genetic adaptation processes during the establishment of chronic pulmonary infections in the CF lung in which (mainly single-base pair change) mutations leading to the loss of function of multiple genes are positively selected [10]. This adaptive behavior seems to be crucial in the development of chronic persistent disease, were $P$. aeruginosa resides in a protected niche within biofilms and hides from the host immune responses. A detailed knowledge on general patho-adaptive mutations will lead to the identification of infection relevant bacterial traits which might be very interesting targets for the development of alternative treatment strategies effective against chronic persistent diseases.

Moreover, since the $P$. aeruginosa microarray presented in this study depicts the whole PAO1 genome including the intergenic regions, the array could also serve as a valuable tool to identify binding sites of transcriptional regulators via the "ChIP-on-chip" technique. ChIP-onchip (Chromatine Immuno Precipitation), is a genomewide location analysis and a technique for isolation and identification of the DNA sequences occupied by specific DNA binding proteins in cells [22]. More than $9 \%$ of the open reading frames in $P$. aeruginosa PAO1 encode for (putative) transcriptional regulators or two-component systems which facilitate efficient adaptation to varied habitats [17]. The identification of the transcriptional regulons involved in the regulation of functions required for bacterial persistence could significantly advance knowledge on specific $P$. aeruginosa adaptation to the environment of the CF lung.

\section{Conclusion}

Chronic $P$. aeruginosa infections remain a major challenge for the medical profession, because even intensified antimicrobial therapy is usually not sufficient to eradicate e.g. persistent infections of the CF lung. Thus, novel anti-Pseudomonas treatment strategies that target the functions required for bacterial persistence are desperately needed. In this study we have developed a robust microarray-hybridization based method that can be applied i) to identify patho-adaptive mutations that facilitate $P$. aeruginosa persistence during chronic infection, ii) as a highly sophisticated bacterial fingerprinting method that may help to identify specific clones or genetic elements that are associated with adverse clinical outcomes, and iii) to identify global regulons by the "ChIP-on-chip" technique that play major roles in the regulation of virulence. These applications should significantly expand our knowledge on bacterial adaptation, evolution and regulatory mechanisms of persistence on a global scale and thus should advance the development of effective antibacterial treatment strategies to overcome persistent disease.

\section{Competing interests}

The authors declare that they have no competing interests. 


\section{Authors' contributions}

$\mathrm{FB}, \mathrm{RG}$ and $\mathrm{SH}$ designed the array in cooperation with Affymetrix. RG performed array hybridization and data acquisition. AD performed the in silico alignment and DNA preparation and analyzed the results. AD and $\mathrm{CP}$ designed software tools for analysis beyond the scope of the Affymetrix software. AD and $\mathrm{SH}$ wrote the manuscript. All authors have read and approved the final manuscript.

\section{Additional material}

\section{Additional file 1}

Java program used for analysis of the PATA1 data. We developed a Java program for post-processing of probe signal and $p$ value data produced by the TAS software (see 'Methods'). The zip file contains a jar archive including program binaries and source code, a sample data set and a readme file with brief instructions for installation and usage. Click here for file

[http://www.biomedcentral.com/content/supplementary/14712164-10-29-S1.zip]

\section{Additional file 2}

Influence of neighbouring nucleotides on sensitivity towards SNPs. For any SNP as identified in the sequence alignment of PATA oligonucleotide probes were grouped by the nucleotides present in both alleles and at the directly neighbouring positions. E.g. 'CGA - T' indicates a mismatch with 'CGA' in PAO1 and 'CTA' in PA14. For each of all 192 possible permutations, 'abundance' indicates how many oligonucleotides showed this particular combination as identified by sequence alignment and 'sensitivity' indicates the fraction of these oligonucleotides that were detected as variant using a threshold signal ratio $D_{t h}=-0.5$.

Click here for file

[http://www.biomedcentral.com/content/supplementary/14712164-10-29-S2.xls]

\section{Acknowledgements}

$A D$ is a recipient of a predoctoral stipend provided by the DFG-sponsored International Research Training Group 'Pseudomonas: Pathogenicity and Biotechnology'. The authors want to thank Jens Klockgether for providing strain P. aeruginosa DSM 1707. Funding from the Helmholtz Gemeinschaft is gratefully acknowledged.

\section{References}

I. Rahme L, Stevens E, Wolfort S, Shao J, Tompkins R and Ausubel F: Common virulence factors for bacterial pathogenicity in plants and animals. Science 1995, 268:|899-1902.

2. Vasil M: Pseudomonas aeruginosa: biology, mechanisms of virulence, epidemiology. J Pediatr 1986, I08:800-805.

3. El Solh AA, Akinnusi ME, Wiener-Kronish JP, Lynch SV, Pineda LA and Szarpa K: Persistent infection with Pseudomonas aeruginosa in ventilator associated pneumonia. Am J Respir Crit Care Med 2008, 178:513-519.

4. Quinn J: Pseudomonas aeruginosa infections in the intensive care unit. Semin Respir Crit Care Med 2003, 24:6I-68.

5. Moreau-Marquis S, Stanton BA and O'Toole GA: Pseudomonas aeruginosa biofilm formation in the cystic fibrosis airway. Pulm Pharmacol Ther 2008, 21:595-599.

6. Tummler B, Bosshammer J, Breitenstein S, Brockhausen I, Gudowius P, Herrmann C, Herrmann S, Heuer T, Kubesch P, Mekus F, Romling U, Schmidt K, Spangenberg C and Walter S: Infections with Pseudomonas aeruginosa in patients with cystic fibrosis. Behring Inst Mitt 1997, 98:249-255.
7. Wagner VE and Iglewski $\mathrm{BH}: \mathbf{P}$. aeruginosa biofilms in $\mathbf{C F}$ infection. Clin Rev Allergy Immunol 2008, 35: I24-134.

8. Romling $U$, Wingender J, Muller $\mathrm{H}$ and Tummler $\mathrm{B}$ : $\mathbf{A}$ major Pseudomonas aeruginosa clone common to patients and aquatic habitats. Appl Environ Microbiol 1994, 60:1734-1738.

9. Romling U, Fiedler B, Bosshammer J, Grothues D, Greipel J, Hardt von der $\mathrm{H}$ and Tummler B: Epidemiology of chronic Pseudomonas aeruginosa infections in cystic fibrosis. J Infect Dis 1994, 170:1616-1621.

10. Smith E, Buckley D, Wu Z, Saenphimmachak C, Hoffman L, D'Argenio D, Miller S, Ramsey B, Speert D, Moskowitz S, Burns J, Kaul R and Olson M: Genetic adaptation by Pseudomonas aeruginosa to the airways of cystic fibrosis patients. Proc Nat Acad Sci USA 2006, 103:8487-8492.

II. Albert TJ, Dailidiene D, Dailide G, Norton JE, Kalia A, Richmond TA, Molla M, Singh J, Green RD and Berg DE: Mutation discovery in bacterial genomes: metronidazole resistance in Helicobacter pylori . Nat Methods 2005, 2:95I-953.

12. Jacobs MA, Alwood A, Thaipisuttikul I, Spencer D, Haugen E, Ernst S, Will O, Kaul R, Raymond C, Levy R, Chun-Rong L, Guenthner D, Bovee D, Olson MV and Manoil C: Comprehensive transposon mutant library of Pseudomonas aeruginosa. Proc Natl Acad Sci USA 2003, 100:14339-14344.

13. BLAST Version 2.2.16. http://www.ncbi.nlm.nih.gov/blast/Blast.cgi.

14. Smith TF and Waterman MS: Identification of common molecular subsequences. J Mol Biol I98I, I47:195-197.

15. JAligner, http://jaligner. sourceforge.net/.

16. Lee DG, Urbach JM, Wu G, Liberati NT, Feinbaum RL, Miyata S, Diggins LT, He J, Saucier M, Deziel E, Friedman L, Li L, Grills G, Montgomery K, Kucherlapati R, Rahme LG and Ausubel FM: Genomic analysis reveals that Pseudomonas aeruginosa virulence is combinatorial. Genome Biol 2006, 7:R90.

17. Stover CK, Pham XQ, Erwin AL, Mizoguchi SD, Warrener $\mathrm{P}$ Hickey MJ, Brinkman FS, Hufnagle WO, Kowalik DJ, Lagrou M, Garber RL, Goltry L, Tolentino E, Westbrock-Wadman S, Yuan Y, Brody LL, Coulter SN, Folger KR, Kas A, Larbig K, Lim R, Smith K, Spencer D, Wong GK, Wu Z, Paulsen IT, Reizer J, Saier MH, Hancock RE, Lory S and Olson MV: Complete genome sequence of Pseudomonas aeruginosa PAOI, an opportunistic pathogen. Nature 2000, 406:959-964.

18. Mathee K, Narasimhan G, Valdes C, Qiu X, Matewish JM, Koehrsen M, Rokas A, Yandava CN, Engels R, Zeng $E$, Olavarietta R, Doud M, Smith RS, Montgomery P, White JR, Godfrey PA, Kodira C, Birren B, Galagan JE and Lory S: Dynamics of Pseudomonas aeruginosa genome evolution. Proc Natl Acad Sci USA 2008, 105:3100-3105.

19. Zhang L, Wu C, Carta R and Zhao H: Free energy of DNA duplex formation on short oligonucleotide microarrays. Nucleic Acids Res 2007, 35:e I8.

20. Vainrub $A$ and Pettitt BM: Surface electrostatic effects in oligonucleotide microarrays: control and optimization of binding thermodynamics. Biopolymers 2003, 68:265-270.

21. Wiehlmann L, Wagner G, Cramer N, Siebert B, Gudowius P Morales G, Kohler T, van Delden C, Weinel C, Slickers P and Tummler B: Population structure of Pseudomonas aeruginosa . Proc Nat Acad Sci USA 2007, 104:8I0I-8106.

22. Ren B, Robert F, Wyrick JJ, Aparicio O, Jennings EG, Simon I, Zeitlinger J, Schreiber J. Hannett N, Kanin E, Volkert TL, Wilson C] Bell SP and Young RA: Genome-wide location and function of DNA binding proteins. Science 2000, 290:2306-2309.

Publish with BioMed Central and every scientist can read your work free of charge

"BioMed Central will be the most significant development for disseminating the results of biomedical research in our lifetime."

Sir Paul Nurse, Cancer Research UK

Your research papers will be:

- available free of charge to the entire biomedical community

- peer reviewed and published immediately upon acceptance

- cited in PubMed and archived on PubMed Central

- yours - you keep the copyright

Submit your manuscript here:

http://www.biomedcentral.com/info/publishing_adv.asp
BioMedcentral 